\title{
Adolescents with Physical Deficiencies - Between Tolerance and Rejection Changing Negative Attitude
}

\author{
Marinela Rusu ${ }^{1}$ \\ 'Researcher, PhD. Romanian Academy, Iasi Branch, Institute "Gh. Zane", Romania \\ Email:marinela1808@yahoo.com
}

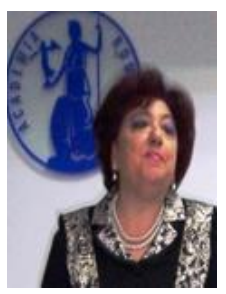

\begin{abstract}
Adolescents with disabilities often face not only the psychophysiological consequences of the presence of physical deficiency but also problems with integration into the school group or in society, in general. The present paper summarizes the conclusions of some researches from abroad as well as the author's regarding the integration of young people with physical deficiencies and proposes a series of intervention methods in order to facilitate this process. Educators are the ones who have an important role to play in changing the negative perception of the deficient person and a better understanding of the concept of deficiency as well. Attitudes of rejection and marginalization are the consequences of social conditions induced by culture, language, history, mass media, etc. All of this can be changed by cultivating counter-values as empathy, acceptance of diversity, tolerance, respect and responsibility. A modern society has to be characterized by humanism, understanding of the other that is different, empathy towards suffering, tolerance and support for those with a deficiency. Our paper is an invitation for educators and social workers to be mindful of changing the negative attitude of young people towards those with disabilities.
\end{abstract}

Keywords: Disability, Rejection, Tolerance, Empathy, Human rights.

Citation | Marinela Rusu (2018). Adolescents with Physical Deficiencies - Between Tolerance and Rejection Changing Negative Attitude. Asian Journal of Social Sciences and Management Studies, 5(3): 123-130.

History:

Received: 27 June 2018

Revised: 13 August 2018

Accepted: 12 September 2018

Published: 27 September 2018

Licensed: This work is licensed under a Creative Commons

Attribution 3.0 License (oc)

Publisher: Asian Online Journal Publishing Group

\section{Contents}

1. Introduction

2. Between Tolerance and Rejection .

Funding: This study received no specific financial support.

Competing Interests: The author declares that there are no conflicts of interests regarding the publication of this paper.

Transparency: The author confirms that the manuscript is an honest, accurate, and transparent account of the study was reported; that no vital features of the study have been omitted; and that any discrepancies from the study as planned have been explained.

Ethical: This study follows all ethical practices during writing.

3. Interacting with Others

124

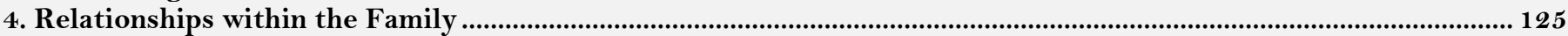

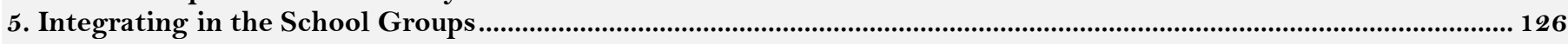

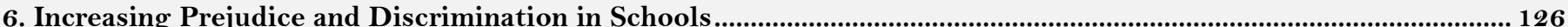

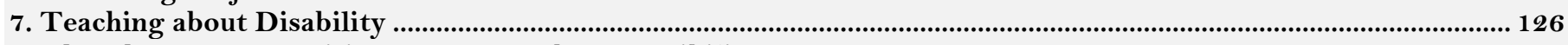

8. The Three R: Recognition, Respect and Responsibility

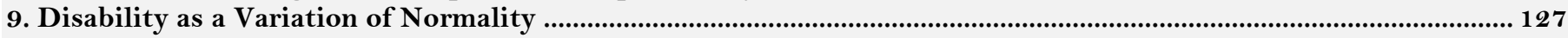

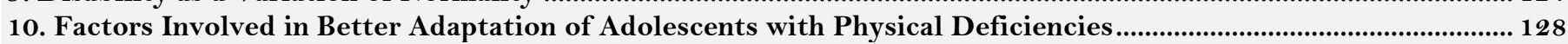

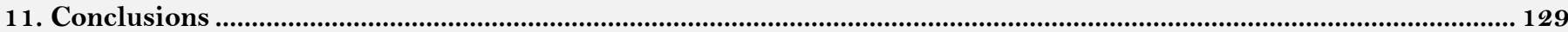

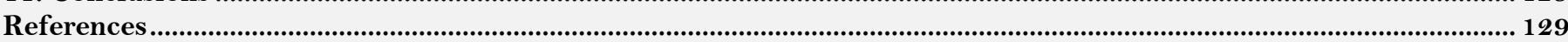




\section{Introduction}

In a world where social prejudices marks largely people's behavior towards their "different" peers, facing us also with its attempt to remove the most harmful of them - such as racial or gender discrimination - the idea of investigating psychological profile of peoples with physical disabilities represents an attempt to enrich knowledge in this area; it is also part of the general effort to remove many of the unfounded ideas about disability and people with a disability. The approach of this theme was sporadic in the Romanian and foreign literature. The groups of people with various physical deficiencies have been the subject of research of limited interest, most often referring to the physiological, medical, rehabilitation and physical therapy aspects. Although we cannot minimize the importance of the medical side, however, we must add the need to consider the psychological issues and their profound implications in shaping the personality and the character. Therefore, the present study proposes precisely a deeper understanding of these human adolescents, who have faced not only the inconvenience of physical deficiency, but must face the devaluating opinions of others, to fight continuously the unfounded doubts about their multiple disabilities, with the idea of personal and professional failure that is mistakenly associated with the presence of physical disability.

Handicapism is still, unfortunately, a truth about the active prejudices in contemporary society. The present study reveals the negative influence and unfavorable consequences of the irrational attitudes of the others towards the persons with physical deficiencies, contributing in the course of the individual development to the crystallisation of disadaptive behaviors, shaping of morbid ideas and self-destructive rumina, generating an inevitable affective fragility.

\section{Between Tolerance and Rejection}

The problem of physical disability is not a new one. It has travelled through the times, generating many popular, mystical and mythological explanations. In many cultures we find associations between images depicting physically deformed beings, and disorders of morality, thinking or conduct. Greek mythology, fairy tales and legends in many cultures describe people with deficiencies as generally unlikeable figures, sometimes even representatives of obscure forces opposing man. Deviations from normal development, not being understood, were attributed to supernatural, fearsome, or repellent forces (Bower, 1980). The Church of the Middle Ages added an ice blast to the difficult state of people with physical disabilities. The fact of not being able to explain how these phenomena appeared meant that they were considered "terrible", signs of evil, manifestations of wrath, divine punishment, and in many cases "dark acts of the devil" (Chauvin, 1920). Even if modern society is making efforts at the legislative level to help people with deficiencies, the collective subconscious is in doubt, emotional cold, and sometimes even hostile to them. Fear of the unknown is a perpetual element of human adaptation that can be overcome only by fearful, penetrating minds and detached from the limitations of forms. Evocators in this sense are the artistic creations, that were accepted by critics as part of the "aura of the ugly". The "ugly" interpreted as "beautiful" become categories that are super ordinated to a reality that can sometimes express identical things. They are not absolute but, on the contrary, as far as human nature is concerned, the relationship between "form" and "content" can sometimes be contradictory: the most appealing face may in some cases hide deplorable characters and vice versa.

\section{Interacting with Others}

Authors such as Păunescu (1983) consider that in the affective and relational structure of the person with physical deficiencies there is a strong conflict state, generating emotional states and negative feelings, which undermine his/her neuropsychic equilibrium. But a conflict cannot be sustained or intensified indefinitely. When the internal pressure exceeds a certain tolerance limit, an exit from the conflict is sought.

Overcoming the feeling of inferiority is often accomplished by the compensation mechanism. As Pirozynski (1987) writes, "as a source of psychic insecurity, the feeling of inferiority gives birth to an ideal of personality, a fiction that reverses the real situation. All the resources of the soul will be mobilized to accomplish it. When acting on the causes of inferiority, compensation is real and the feeling of inferiority dissolved. But there are also situations where, for subjective or objective reasons (frustrating social conditions, etc.), one can only act on the effect (i.e., the feeling of inferiority). Then we have a pseudo compensation: its means are imaginary-subjective; the feeling of inferiority is not dissolved but it is pushed into the unconscious." In the same sense, Rudica (1992): "no less frequent and important through its consequences, is that category of vocational frustrations that are caused by various motor or psychic infirmities that make those adolescents quit the dream of a particular profession, with the conscience and regret of forced abandonment. Some of these disabled people are developing successful compensation phenomena along the way, which help them form a new professional ideal, but others fail to balance through other activities and remain for all their lives with the painful feeling that nature has deprived them of the chance to do things the way they want." In the opinion of Păunescu (1983) there are two major aspects of this process: "the first will lead us, he writes, in a general way, out of the conflict that puts the question of changes in personality" (p. 20). In establishing friendly relationships with others, the building of self-image has a particular importance. Self-image is a dynamic synthesis in which the others reflect, and also reproduces the condition of our own existence. Due to the presence of a "weak point" that is the deficiency, felt by the subject directly, but also through the attitude of the others towards him, he tends to report everything and especially his affective-emotional feelings, to his physical ego, acquiring a special, excessive "sensitization" (Rusu, 1996).

The affective level is best suited for installing this sensitivity. "Exploring your own body" (Schwarzer, 1992) is a behaviour that runs from the first year of life to adulthood, allowing individuals to discover and learn their own body as a differentiated entity, unique to each of them. The discovery made by the person with deficiency is that this "uniqueness" has a negative sense and often leads to opposing, tense experiences. Both objective and affective knowledge receive the disfavouring situation more clearly. "Even at an early age, the child has to deal with deficiency as something special and denigrating, because of the discussions between parents or between adults; after that the deficiency becomes a whip with which the other children hit and humiliate him/her" (Rusu, 2002). It 
is a simple fact to demonstrate that children's attitudes are influenced by general educational attitudes, by the attitude of adults towards people with physical disabilities.

\section{Relationships within the Family}

Automatically become mirrors in which the young person can look and understand himself. Some specialists claim that parents create the deficient socio-emotional status through the relationships they create in the first stage of the child's life, parental "mirrors" helping the process of self-defining. Trying to establish a typology of relationships within the primary group where there is a child with physical deficiency, we will identify the following types: Total affectional abandonment - since the discovery of the deficiency, parents cannot admit the failure suffered by the appearance of a child that puts them in inferiority in their community. In this case, the child's suffering will be complete and its development slowed down. Partial abandonment-occurs when one of the parents especially the father - is completely careless concerning the child's needs. The overwhelmed mother becomes emotionally aggressive with the child, traumatizing him. Mother has a feeling of condemnation in front of destiny and can have two distinct attitudes: either completely abandoning the child, or making a total affective fixation towards the child, completely giving up her personal life. There are cases when the existence of a child with deficiency leads to a hyper protective attitude in parents, for example, the manifestation of an exaggerated worry towards the child, suppressing his/her possibility of becoming autonomous. Unfortunately, the most common situations are those in which a deficient child breaks the family's balance, through strong conflicts stemming from the mutual blame of spouses about their "misfortune" (Rusu, 1995a;1995b).

Any of these situations is detrimental to the development of the personality of the deficient child, because the stimulation of affectivity, activity, relationships, tendencies to manifest autonomy, independence are the essential means for the development of a harmonious and integrated personality. So, the way the family responds to this accident - which must be considered as any other accident - depends on the structure and the development of their own personality. Meisel (1986) notes in this regard: "A group of parents who came regularly to the consultation showed their intense suffering and the terrible emotional distress caused by the infirmity of the child. Some thought their marriage had suffered, others were afraid they had neglected their other children. Many felt the inability of friends, relatives or neighbours to understand and accept their own child. In others, the greatest despair came from the lack of hope: will their child continue to develop mentally and physically? Many talked about resentment to the professional world that advised them to place the child in an institution or against those counsellors who fail to understand them." The attitude of hyper protection seems to be, often, as a materialization of a sense of guilt. It can have a disastrous effect on child rehabilitation. For the disabled child as well as for the normal individual, nothing is more convenient than the state in which it is not obliged to make any effort, everything being driven, carried out by others. Only that, starting from here, creates even worse infirmity. Even that child, who, manages to become independent, with respect to motricity became capable of self-service, in cases of hyper protection becomes a complex infirm, insecure of his abilities, waiting everything from others. He becomes lonely and later will consider his condition an injustice and will firmly demand that society take care of him when his mother is gone, not conceiving that he may be able to help himself. He becomes a lazy, often evil, vindictive teenager, who easily adds up vices of all kinds. The complexity of disability manifestation let us identify another important aspect concerning the adolescents with deficiencies: the relational handicap or the issue of social contact. Relating to normality is for these adolescents a continual reaffirmation of infirmity, generating the feeling of embarrassment, inferiority, fear of not being accepted, and often they repel the attitudes of repulsion, rejection of an intolerant society with its marginalization tendencies.

With constant attention and constant dialogue, we discover the immense anguish of these children for whom ordinary accents or words gain a considerable, delusional, fantastic importance and they are moving away from those who do not know them very well. Next, we will try to delineate the importance of the concept of relational handicap, somewhat extending its sphere of comprehension, in the case of adolescents with physical deficiencies. We will complete the definition given above by C. Păunescu, showing that relational handicap is an impossibility of the ego to project beyond infirmity and to creatively assume the environment in which the teenager lives. As we observed in our experimental intervention with these children, some years ago, "The relational handicap is at the same time the reflex of a false identification, following the illogical equation, the unreliable me $=$ my infirmity, me $=$ my failures, me = the helplessness" (Rusu, 1995b). If in the case of severe psychoses the hope of healing relies on therapeutic action on the still unaltered part of consciousness, similarly, in the case of relational disability, we must count on the "unaltered" elements of the adolescent personality. And these "unaltered" elements refer to the mechanisms of thought, logic, and to the same extent to general human truths. The relational handicap is mirrored within the personality by a tendency dominated by repelling and avoiding social contact. This measure of defense of the personality balance results on the part of the weak cultivation of the mechanisms of relationship, of intercognition, and above all, of an over amplifying the normality of others, simultaneously with the over amplification of their own infirmity. Particularly serious may be the effects in time of relational handicap manifestation. It can produce fragile personalities, irreversible or hard-to-recover affective perturbations. The mechanism known as self-fulfillment prophecy (Schwarzer, 1989) becomes here a suitable example. It consists in the tendency of a person to become what he is expected to be or what is believed to be about. The ability to establish normal relationships with others is virtually the only saving valve in the face of the gradual dissolution of a person touched by the inferiority complex. The balanced, natural relations are the only ones capable of resizing a correct selfimage, in which $\mathrm{I} / \mathrm{me}$ is not equivalent to the deficiency, in which the deep self does not identify with personal failures but possesses a tremendous potential for internal restructuring and evolution. Also, only normal relationships with others can restore authentic hierarchies of individual values, removing the idea of "stigma" or "damnation." In this way, the process by which relational handicap would turn into a psychosocial disability can be prevented. Jealousy and autism are two reactions to the hostile environment and two facets of the relational handicap. Autism, closure itself, refusal to collaborate is in essence a trick of pseudo-learning of deficiency, because autism actually means to see without being seen. Jealousy is a social, but negative attitude, and not a few times it can become a sadistic attitude. But there are different types of characteristics. There are children who are looking for 
people's society and cannot live outside a circle of friends, even if the feelings that unite them are not really close. Others choose isolation and are content with a circle of restricted friends whom they have been attached to for a long time. Friendship creates the possibility to exchange impressions or feelings that one can only tell about to a good friend, expressing the embarrassment about the issues that troubled one's teen years. Some relationships of this kind are irreplaceable for the psychic and moral life and evolution of adolescents with physical deficiencies.

\section{Integrating in the School Groups}

Schools have a responsibility to change negative attitudes that lead to discrimination, prejudice and segregation. Because it is precisely a central goal of education, promoting the values and attitudes that are essential to our pluralistic society and to the democratic form of government, it is particularly important to refer to those beliefs and attitudes that lead to discrimination - the denial of certain members of society, the right and opportunity for full participation at the social, educational, economic and political levels. Therefore, educators need to understand the importance of teaching future citizens the care for the rights of others (Lickona, 1991). Schools can play an important role in bringing about the necessary social changes, preparing for "each generation to examine and, when necessary, to alter existing social values, practices and beliefs" (Shapiro, 2000). The drive to reintegrate young people who have been excluded on the basis of disability is an "exciting, controversial and dynamic process for provoking the basic assumptions of each of us and at the same time introducing us in new ways to think and see" (Biklen and Bogdan, 1977).

A school has the responsibility to contribute to the socialization and unification of society. As Rubenfeld (1994) stated, school, of course, is one of the great defining experiences in any country. Almost more than family life, it is the "Great Socializer". Here we learn to juggle with friends, enemies and knowledge, to cope with difficulties and to enjoy success in our projects.

\section{Increasing Prejudice and Discrimination in Schools}

Contrary to common belief, there are considerable damages among adolescents which tend to grow and become more aggravated as the years go by Baskin and Harris (1977) found that young people classified as handicapped are often rejected and ostracized by their peers. In situations of high density, potentially explosive, such as dining rooms or playgrounds, they have been particularly vulnerable to social abuse. "Simply, students with disabilities are often not chosen as friends" (Kelly and Reigel, 1993). "While some studies suggest that disabled students perceive their peers in general, positive ... most studies indicate that non-disabled students show negative attitudes towards their peers with deficiencies" (Pfeiffer, 1989). It is not surprising that Lickona (1991) claimed cruelty among colleagues as one of the scary trends in schools, along with a sharp rise in violence and vandalism, lack of respect for authority, bigotry, mischievous language, and lower civic responsibility.

\section{Teaching about Disability}

According to Orlansky and Heward (1981) the main factors that form the development of negative attitudes are the media, which tends to negatively affect people with disabilities. Bowe (1978) pointed out that as long as society fears people with disabilities, they will continue to treat them in hospitals, institutions and separate schools. He wrote: "As long as we compassionate people with disabilities, we will continue to regard them as objects of charity rather than equal in order to have a say in their destiny" (p. 111).

\section{The Three R: Recognition, Respect and Responsibility}

Recognition is about admitting the truth that there is diversity in life and each individual is different and has this right, implicitly. To recognize the difference also means the ability to respect this difference and to take it into account in interpersonal interaction.

Respect refers to the means by which someone demonstrates respect for the value of someone else. Respect for others requires an individual to respect all other people as having equal dignity and equal rights with one's own person.

Responsibility is an extension of respect. In the Lickona (1991) if we learn to respect people with disabilities, it also means we value them. If we value them, we feel some responsibility for their well-being. Educators and legislators freely recognize the benefits of integrating young people with educational disabilities with their nondisabled colleagues.

Before discussing such educational and social benefits, we still need clarification of the terms. The concept of "integration" involves several terms that confuse those who do not know their specific nuances. The best-known terms are (1) "the least restrictive medium" (or alternatively), (2) "normalization", (3) "integration" and (4) "inclusion".

Integration simply refers to the concept of group homogeneity. It is the direct opposite of segregation, especially in homogeneous environments such as special schools, special institutions, separate classes and services" (Bowe, 1978). Integration typically refers to the process of returning students with disabilities to general education, while maintaining a separate system of special classes, services and programs for others. Attitudes can be changed. As young people with harmful behaviors become aware of the effects of negative feelings, they can develop the desire to change. Since attitudes to young people are learned, the cognition theories can be applied to change negative attitudes. As young people receive new ideas and incentives, their prejudicial attitudes often fade away: "Attitudes appear to be highly dependent on contact and information about what specific attitudes are being raised" (Bowe, 1978). But the settings of young people's contacts with disabled people are extremely critical. When a young person with disabilities integrates into the ordinary school, all children benefit from both a better learned curriculum and the opportunity to learn to accept and appreciate individual differences. Teaching methods, such as cooperative learning, improve and enhance the academic abilities of young people, since we need to learn to educate. 
This paper provides information designed to change the negative attitudes of teachers and students to develop empathy for people with disabilities and to support their inclusion in both school and society. Changes in attitudes and the development of empathy can help to overcome the gap between people with disabilities and those without. The process begins by studying and acquiring a complete understanding of the paradigms of special education and disability, especially the medical model and the model of independence. Students and teachers need to learn that disability does not focus on the medical causes or etiologies, but on understanding the negative effects of the social and civic environment on disability. Its favorite idea is that it is possible to reduce the number of people with disability discrimination in schools by eliminating negative attitudes and segregation that comes largely from the medical paradigm. Blatt (1987) emphasized that conquering the mental retardation and eliminating its rejection would only be achieved when young people learn to live with each other and until the separation of all forms is eliminated, until schools not only believe but teach indeed that all human beings are precious and valuable to society.

Meanwhile, to get empathy and true classroom integration, teachers need to know the following aspects related to the issue of deficiencies (according to Shapiro (2000)):

1. To identify those elements which contribute essentially to the emergence of negative attitudes, including: language, history, culture, the media and education;

2. Re-evaluate the impact of language and the meanings of the concepts of "disability", "handicap", "deficiency", "stereotype", "prejudice" and "discrimination";

3. To find methods of developing pupils' awareness of the deficiency and its relation to other forms of discrimination, in particular, the similarities and differences between racism, sexism and deficiencies;

4. Use specific methods that lead to successful integration;

5. To find methods that can be used to assess pupils' negative attitudes so that these attitudes can be changed;

6. Strategies and methods - for example, simulations, role plays, classroom discussions, interacting with deficient people (their presence in the classroom, dialogue) and attending a documentary hour with media sources - to facilitate individual differences learning;

7. Specific teaching strategies and methods to dispel popular beliefs and myths about people with disabilities - for example, these people are sick, they prefer and need separation, they need and seek sympathy, they are similar in groups of people with disabilities, are abnormal or less valuable;

8. Find ways to sensitize students to understand and act with the frustrations and restrictions imposed by the different sensory, physical or mental conditions;

9. How to provide opportunities for teachers and students to share experiences and feelings about the condition of being disabled and disabled in a favorable group environment;

10. How to give young people the opportunity to reflect on their feelings when they are with a disabled person;

11. How to defuse the invisible barriers of fear and curiosity that teenagers can have about people with disabilities, especially classmates and /or deficient guests;

12. How to help young people develop awareness and appreciation of what it means to have a disability;

13. How to help students understand the potential and limitations of corrective devices used by people with disabilities;

14. How to help young people see beyond the disability the people who are, in reality, more akin to them than different;

15. How to improve the ease with which both students interact with people who seem different, no matter what difference might be;

16. How to provide basic information on common disabilities needed to develop empathy and to shape a positive outlook towards people with disabilities;

17.Recognizing the way of examining your own thoughts and feelings towards people with disabilities;

18. How to make students understand that disability denies humanity to both people without disabilities and those with disabilities;

19. Making students understand how the physical and social environment can be improved so that disability should not be perceived as a handicap;

20. To help students understand the civil rights of people with disabilities.

\section{Disability as a Variation of Normality}

The use of the term "normal" to refer only to individuals without disabilities means the involvement of those with "abnormal" disabilities (Blaska, 1993). "Abnormal" has extremely negative implications. It is offensive and should not be used when referring to human beings. Educators must accept the concept of all people, including those with disabilities, as normal. In other words, all people should be perceived as living in a very diverse group, where all are normal and equally worthy.

We recall that White (1979) insisted on the idea that we cannot speak of similarity, identity, normality (in the typical pattern, template) in the case of human beings. This is confirmed by the diversity of conformations of individual features, qualities or decreases that favour success or adaptive failure. In some philosophies of readaptation, it is presented that instead of normal behaviour - as the gold guide says for "what to do and how to do" the abilities and deficiencies of a person will be all put into action, and thus the person will be really fulfilled. Here is what we find written in the booklet dedicated to patients at the Institute for Physical Medicine and Rehabilitation in England: "There are hundreds of ways to do all this to help us become independent. Maybe with your deficiency, you will not be able to do it the way others do, but it certainly does not mean that you cannot learn to do it in a different way. That's why our people are here to teach you to do these things as best as possible with your deficiency" (as quoted in Allen and Pearson (1988). 


\section{Factors Involved in Better Adaptation of Adolescents with Physical Deficiencies}

Following the findings in our experiment, which started some years ago (Rusu, 1995a;2002) concerning the adaptation of adolescents with physical deficiencies, we were able to identify a number of factors that allow better adaptation and social integration of these teenagers.

In addition to psychological factors that can contribute to personality optimization, there are certainly a number of environmental and social factors that favour a better adaptation of adolescents suffering from physical deficiencies, leading to the emergence and development of compensatory behaviours. Authors such as Orlansky and Heward, who have studied closely the issue of optimizing social adaptation of people with disabilities, agree on a number of favourable factors, including the following:

\section{Functional Recovery of the Person with Deficiencies}

This will be achieved by the combined efforts of surgeons with those of physiotherapists, to correct the physical defect as much as possible or totally. Reclaimed independence consists in the use of such material means (wheelchairs with automatic controls, moving racks, crutches, prostheses), which make changes to the environment (incoming walk-in ramps, special baths, intercoms, etc.) that make it easier for these adolescents to move and minimize dependence on others.

Social contacts are the area that brings attention to the issue of reinsertion into a micro group with a secure climate for teenagers with physical deficiencies. In this respect, we recall here some rules of self-control of curiosity towards people with deficiencies, which should be observed within the group (according to Wright (1960) do not talk about deficiency unless he/she (the person with deficiencies) does it; do not talk about deficiency unless he/she wants it; do not ask immediately; take into account the person's condition; do not argue on this subject; do not try to divert it from the subject.

\section{Personal Valorisation of Adolescents with Physical Deficiencies}

Here we may include a large number of suggestions and concrete proposals. Some general directions in this respect would be:

a) accepting the deficiency - this requires awareness of the limits while recognizing the skills and abilities of adolescents with physical deficiencies. Thus, they will end up accepting their own limitations, cultivating their own abilities, and concentrating their efforts to reach accessible goals. For this, it is necessary to outline a self-concept that includes both positive and negative attributes, being preferred to the belief that they possess either an exaggerated powerlessness or an imaginary omnipotence. It is also necessary to strengthen feelings of adequacy and trust based on the current personal possibilities to act in the social, educational and vocational fields;

b) Achieving and maintaining expectations of performance and fulfilment. Comparisons with other children will be avoided and appropriate standards will be set for individual potentials. Expecting more from a teenager with deficiencies can lead to frustration, a sense of failure and inadequacy. Also, waiting too little of them often leads to an exaggerated dependence, as well as a feeling of weakness and resignation;

c) supporting and encouraging the teenager's urge to become independent. People with deficiencies in general, are in a position to remain addicted to others much more. That is why the greater the number of independent activities they perform on their own, the lesser their shortcomings will be.

d) creating varied experiences in the areas where the teenager is able to fulfil. These will be encouraged by teachers or parents.

e) Establishing appropriate discipline requirements, as physically deficient adolescents require much improved school and living space.

Two specialists have an important role in the formation of children and adolescents with physical disabilities are physiotherapist (dealing with physiological recovery) and occupational therapist who seeks the participation of adolescents in various activities, especially those which are useful for self-help, employment, communication and other aspects of life (Baskin and Harris, 1977). Such activities foster physical development, independence, vocational potential and self-image enhancement.

The occupational therapist can also work with the vocational rehabilitation specialist to help teenagers find those opportunities to work and live independently after school. Orlansky and Heward (1981) calls also other specialists who frequently offer help to adolescents with physical disabilities, such as: physical therapist or physiotherapist - he is encouraging children to become as independent in terms of motility, which helps to develop motor functions, to reduce pain, discomfort or long-standing problems related to physical impairments; biomedical engineers who create and develop specialized technology to meet special needs; a nursing social assistant that helps deficient students and their families to cope with difficulties; analysts of practical behaviour that closely follow the behaviour of adolescents with physical deficiencies. In fact, we can talk about the possibility of building a team of specialists to collaborate directly on physically impaired patients.

\section{Social Valorisation of Adolescents with Physical Deficiencies.}

Beatrice Wright (1960) addresses an important problem concerning how the deficient person relates to the "world" of others. She noticed that she often has inappropriate reactions in uncomfortable and devaluing situations. Thus, a person who is always confronted with difficult situations will gradually develop ways to overcome these situations. Her approach to the group will depend on general self-assessment. As a result, a form of therapy has been approached, aiming at better adaptation to social situations created under different circumstances. Role Playing is the method by which a teenager with a physical deficiency learns to give the best answer, to have the most appropriate conduct in the face of curiosity, insult, insensibility of people without disabilities. These modes of behaviour are designed in situations where guidance and encouragement are possible and where there is control over mistakes. The discussion about the following personal experiences is completely free, which produces over time changes in the personal system of values. It is an extremely useful method to overcome one of the most serious problems of the deficiency: relational handicap. More confident about what he is and what he can do, the 
physically deficient adolescent meets the demands of the environment altogether, and he is more committed to fulfilling his professional aspirations.

Another interesting idea in this respect is the reintegration of these children into the classes of children without disabilities. In his article Lovitt (1979) mentions that: "Of course, it is not easy for teachers, but they will gradually become accustomed to the somewhat different needs of children with physical disabilities, so they will be eventually treated like their other colleagues"(1979). Many adolescents with physical impairments manage to adapt to their own living environment. They do not have unusual behaviour and are fully capable of learning in classes with physically normal students, interacting harmoniously with them. In the same sense, Meisel (1986) observes that "as the view that people with physical deficiencies need to depend on assistance for their education, is being overcome, in the same way, there is a question mark over the opinion that medical establishments and medical staff are the ones more appropriate to coping with academic learning, day-to-day skills, or social behaviour of deficient adolescents"(p. 87). Current treatment methods make it possible for many children with deficiencies to attend regular schools. Hospitalization tends to be shorter, physiotherapy can be done even in school and surgery can often be done during holidays.

In conclusion, we have to revert to the idea that people with deficiencies develop their own style depending on certain needs imposed by deficiency. But their inner feelings tend to be the same as the others'. We should understand that they do not want and do not need pity, compassion, but only minimal help, detached, but above all they need to be considered as all others, human beings who aspire to happiness, personal and social fulfilment.

\section{Parents - The Most Important Element.}

Of all people who need to help children with physical disabilities, parents are the most important. Knowing all the details they will help prevent many of the risks that may arise. In many Western countries, parents have legally acquired the opportunity to make decisions about the educational option for their children with physical or mental deficiencies. Both in terms of providing assistance and training, parents can help their children with difficulties and even teach them at home or at school. It is now known that the most successful intervention programs involve the intervention of parents. Shearer and Shearer (1972) confirm that parents engaged in early intervention programs - at young ages - for children with various deficiencies took the role of members in counseling boards, consumer roles that inform others, organizational members, teachers, curriculum developers, counselors, support staff, or assessors. Parents are the most frequent and constant observers of their children's behavior. Often, they know better than anyone what are the adaptive needs of their children and they can help educators in setting realistic goals. They can also tell stories about home events that cannot be captured by outsiders - for example. Parents always know best how the child reacts to other family members, to the censored environment of the school or medical center. In short, parents can take part in rehabilitation programs - integration involving their children at any stage - setting up, planning, and participating in classroom activities and assessment. Many parents work in classes as teachers, teacher help, as volunteers, or as members of organization and leadership. This is true both in childhood and adolescence, when the problems of the child become more complex, and it is necessary to orientate him towards a profession and to pay attention to the individual vocation. Trust and optimism should be the dominant notes in parent-adolescent relationships.

\section{Conclusions}

Looking at the problem in a temporal perspective, we would say that the deficient young and adults have a permanent problem: the lack of acceptance as fully integrating members of society, with all the rights, privileges and services guaranteed as for every citizen. Even though the law has progressed somewhat, even though justice has laws in favor of these people, it can be quite difficult to change the way some individuals think or deal with people with shortcomings. The latter "appear to exist in the community but not to be part of it" as Birenbaum (1986) writes. In this respect O'Conner notes an impressive fact: "we should remember that there is really little evidence to date that public support for normalization has gone so far that people with mental deficiencies are welcome or at least tolerated in the most no deviated circles of society" (as quoted in Heward and Orlanski (1988). Most young people and adults with physical deficiencies point out that the greatest barriers to total integration into society are not the inaccessible buildings or current restrictions imposed by their deficiency, but the differential treatment given by persons without deficiency. As terms like racism and sexism indicate prejudice, discriminatory treatment of racial or women groups, the American term "handicapism" (Shaver and Curtis, 1981) has been designed to mark special responses to people with disabilities. Such reactions are not based on the real qualities or performance of the individual, but start from the assumption of what a person with a disability should feel or how it should be as a result of his or her disability.

All the efforts are oriented toward avoiding this destructive attitude to adolescents with physical disabilities. The factors that we presented above are important to take into consideration when trying to optimize the perception of deficient persons, when searching for a better adaptation and integration. School and teams of specialists can also give their specialized help, working together with trust and optimism.

\section{References}

Allen, F. and C. Pearson, 1988. The emotional problems of the physically handicapped child. British Journal of Medical Psychology, 8: 56-63.

Baskin, B. and K. Harris, 1977. Notes from a different drummer: A guide to juvenile fiction portraying the handicapped. New Y ork: R. R. Bowker Company

Biklen, D. and R. Bogdan, 1977. Media portrayals of disabled people: A study in stereotypes. Interracial Books of Children Bulletin, 8(6 \& 7): $4-9$.

Birenbaum, A., 1986. Community programs for people with mental retardation. Mental Retardation, 24(3): 145-146.

Blaska, J., 1993. The power of language: Speak and write person first. In perspectives on disability (2nd Edn.,). M. Nagler (Ed.). Palo Alto, CA: Health Markets Research.

Blatt, B., 1987. The conquest of mental retardation. Austin, TX: Pro-Ed. Inc.

Bowe, F., 1978. Handicapping America: Barriers to disabled people. New York: Harper \& Row.

Bower, E., 1980. The handicapped in literature. Denver, CO: Love Publishing Company.

Chauvin, E., 1920. Accuracy of teratology. Paris: Masson et Cie, Publisher. 
Heward, W. and M. Orlanski, 1988. Exceptional children. Ohio: Columbus.

Kelly, F. and D. Reigel, 1993. Teaching the student with spina bifida. Baltimore, MD: Paul Brookes. pp: 245.

Lickona, T., 1991. Educating for character. New York: Bantam Books.

Lovitt, T., 1979. What should we call them? Exceptional Teacher, 1(1): 5-7.

Meisel, J.C., 1986. Mainstreaming handicapped children: Outcomes, controversies, and new directions. Hillsdale, NJ: Lawrence Erlbaum Associates.

Orlansky, M. and W. Heward, 1981. Voices: Interviews with handicapped people. Colombus, OH: Charles E. Merrill Publishing Co.

Păunescu, C., 1983. Poor child - knowledge and education. Bucharest: Scientific and Encyclopedic.

Pfeiffer, D., 1989. Attitudes, disabled people and the law. In S. Hey, G. Kiger, and D. Evans (Eds.), The changing world of impaired and disabled people in society. Salem OR: The Society for Disability Studies and Willamette University. pp: 5-17.

Pirozynski, T., 1987. Psychiatric assistance in cases of pathological behavioral risk. Iasi: Clinic of Psychiatry, Junimea Publishing House.

Rubenfeld, P., 1994. Special education: An institution whose time has come and gone In E. Makas \& L. Schlesinger (Eds.), Insights and outlooks: Current trends in disability studies. Portland, ME: The Society for Disability Studies. pp: 235-238.

Rudica, T., 1992. Mature personality. Iasi: Junimea.

Rusu, M., 1995a. Conductive compensators for physically deficient adolescents, in rev. Special Education(1): 12-18.

Rusu, M., 1995b. Compensation - revealing element in individual adaptation, Psychology between tradition and innovation, A. Munteanu (Ed.), Timişoara: Mirton. pp. 232-239.

Rusu, M., 1996. Anxiety - self-consciousness and expectancy. Journal of Psychology, 8: 17-23.

Rusu, M., 2002. Affective life and compensation. Bucharest. Rowley: Sapientia Publishing House

Schwarzer, R., 1989. Current trends in anxiety research in European perspectives in psychology. Chichester: Wiley, 2.

Schwarzer, R., 1992. Self-efficacy as a resource factor in stress appraisal processes self-eficacy, thought control of action. Washington: Hemisphere.

Shapiro, A., 2000. Everybody belongs: Changing negative attitudes toward classmates with disabilities. New York: Routledge Falmer.

Shaver, J. and C. Curtis, 1981. Handicapism and equal opportunity: Teaching about the disabled in social studies. Reston, VA: The Foundation for Exceptional Children.

Shearer, M.S. and D.E. Shearer, 1972. The portage project: A model for early childhood education. Exceptional Children, 39(3): 210217.Available at: https://doi.org/10.1177/001440297203900304.

White, B., 1979. Understanding persons with disabilities. Lincoln, NE: Lincoln Public Schools.

Wright, B., 1960. Psychical disability. A psychological approach. New York, Evanston: Harper and Row. 